\title{
IAU Symposium 266: Summary
}

\author{
Giovanni Carraro \\ ESO, Alonso de Cordova 3107, 19001, Santiago de Chile, Chile \\ email: gcarraro@eso.org \\ Dipartimento di Astronomia, Università di Padova, Vicolo Osservatorio 5, I-35122 Padova, \\ Italy \\ email: giovanni.carraro@unipd.it
}

\begin{abstract}
A review is given of the topics discussed during IAU Symposium 266 on star clusters, and of the major results presented. The current state of the art is discussed, together with potential prospects which may help to direct and generate future advances.
\end{abstract}

Keywords. globular clusters: general, open clusters and associations: general, Galaxy: halo, Galaxy: disk, Galaxy: bulge, stars: low-mass, brown dwarfs, Hertzsprung-Russell diagram, stars: horizontal-branch, binaries: general, stars: abundances

\section{Introduction}

IAU Symposium 266, Star clusters: basic galactic building blocks throughout time and space, hosted 47 talks including the plenary review and summary talk. As the title emphasizes, star cluster studies currently cover a large range of topics in Galactic and extragalactic astronomy. In fact, star clusters are laboratories of stellar evolution and dynamics, as well as building blocks facilitating studies of the properties of external galaxies, including their cosmological formation and assembly, star-formation history (SFH) and chemical evolution. In this summary, I report on the diverse and rich material discussed at the meeting, grouping together the various talks where appropriate.

\section{Star clusters and star formation}

Star clusters are ideal sites to study the formation of stars in a statistical way. Researchers at the meeting agreed that stars form from dense $\mathrm{CO}$ cores inside molecular clouds. In addition, (almost) all stars form in star clusters and tend to group together in hierarchical structures. This model emerges and acquires further confirmation from recent and ongoing extensive submillimeter, infrared, optical and X-ray surveys, both photometric and spectroscopic, which were presented during the meeting. The major role that the Atacama Large Millimeter Array will play in this context was emphasized, particularly its unprecedented spatial resolution at these wavelengths.

The study of very young clusters or associations can help us probe the first phases of the star-formation process and the complex interplay between stellar evolution, feedback and interactions. A large variety of stellar systems exist, from nuclear star clusters to associations, and from embedded clusters to super star clusters, such that the starformation process is actually studied in very different environments. From detailed X-ray studies, evidence was presented of clusters having a sequential $\mathrm{SFH}$, while others show prolonged SFHs. 
At some point, we should correlate these findings with the dynamical status of clusters and their location in the Galaxy, to see whether star formation is an environmentally dependent process.

\section{Star clusters and stellar evolution}

This is a classic topic in astronomy and, as usual, observations offer interesting news and challenges, which in turn motivate continuous revision of the theory. For instance, the discovery and characterization of the intriguing multiple stellar populations in Galactic and Large/Small Magellanic Cloud globular clusters (the commonly believed standard example of single stellar populations) through extensive photometric and spectroscopic campaigns is boosting major theoretical efforts.

We need to understand how this complicated mix of populations formed, in essence how clusters could retain processed material from first-generation stars to produce second- or even third-generation stars. The competing roles of rotating massive stars and evolved stars on the asymptotic giant branch were discussed in this context, but without reaching clear-cut conclusions. Alongside this discussion, a new scenario in which the gas necessary for the second generation is expelled from first-generation binary systems was proposed and attracted much interest.

The enhanced/nonenhanced helium-abundance explanation for secondary blue sequences in the color-magnitude diagrams is another hot topic, and a clear example that there must be some missing ingredient in our current understanding of stellar evolution. Thus far, researchers have mainly concentrated on the internal evolution of star clusters, but it might be worth to take a look at completely different scenarios, such as external perturbation and minor mergers. At any rate, we need to pose the fundamental question: Can we still consider globulars as simple stellar populations?

Studies of brown dwarfs in young clusters, helium-burning stars in the horizontalbranch phase, blue stragglers and white dwarfs in intermediate-age and old systems were presented. These will potentially modify several fundamental aspects in our understanding of stellar structure and evolution (e.g., mass limits, lifetimes, the role of binaries) in the near future.

\section{Star clusters in external galaxies}

The study of star clusters in external galaxies seems quite lively. Here, star clusters are mostly considered test particles, and their integrated properties are used to deduce general properties of galaxies. Quantities such as luminosity and mass functions, age-mass relationships and color distribution are derived to determine the initial mass function (IMF), the SFH and (more generally) the cosmological formation scenario (either monolithic or hierarchical) of the host galaxy.

Critical issues related to measuring these quantities were discussed, like limitations of the integrated photometry, proper filter selection and binning techniques, as they might interfere with the objectivity of the basic measurements and the resulting conclusions. This is illustrated by the intriguing situation of using completely different approaches for deriving star cluster luminosity functions which produce basically the same results! It was argued that better communication among researchers and exchanges of processed data can help solve many of these conflicts.

In various models, star clusters are the building blocks of galaxies and the interaction between clusters and their environment is investigated to study both cluster evolution 
and the formation of structures such as bulges. The role of the tidal field, disk shocking and dynamical friction was discussed in unprecedented detail.

\section{Star cluster formation and internal dynamics}

Many models of the formation, early evolution and dissolution of star clusters were discussed during the conference. Feedback from massive stars and their interaction with the environment, giant molecular clouds and the host galaxy's potential well determine how long a cluster with a given initial mass can survive, and possibly when mass segregation occurs. This has been observed to take place at different times in different clusters, but it is not yet clear whether or not mass segregation occurs at the very beginning of star cluster evolution.

The role of the IMF and its universality was discussed in this context, together with the never-sufficiently-stressed importance of binary stars, white dwarfs and black holes. Dedicated proper-motion and radial-velocity studies allow us to pin down actual cluster members and provide more solid estimates. Discrepant results were shown, casting doubts on the reality of black holes in clusters. The IMF was shown to be the same in very old and very young clusters, supporting is possible universality.

$N$-body models are getting more and more sophisticated, and we heard that the coupling of stellar evolution with dynamics making rapid progress, although significant differences in the results from different codes are still present. However, $N$-body and stellarevolution models combined already show an impressive level of detail and a remarkable similarity to actual observations for some individual clusters (notably M67 and NGC 188).

\section{Star clusters in the Milky Way}

The structure and chemical evolution of the Milky Way and its components have traditionally been investigated using star clusters. The parallel IAU Symposium 265 on chemical abundances discussed many of these classical topics, which had only minor coverage during our meeting.

Globular clusters are used to probe the formation of the Galactic halo, and very detailed chemical studies attempt to clarify whether stars floating in the halo originate from globular-cluster mass loss. Isotopic abundances are leading to completely different opinions, which hopefully will converge when more data come available. Opposite claims are, in fact, based only on a limited number of clusters.

The origin, age, shape and metallicity distribution of the Galactic bulge was also addressed using globulars, in spite of the difficulties that the high and patchy reddening poses. The Galactic-disk spiral structure and chemical evolution is another traditional topic, where progress will benefit from the intense hunt for highly obscured, overlooked star clusters. Still, new spiral features have recently been discovered in the outer disk, mostly in the third Galactic quadrant. Hopefully, some other massive young clusters - so rare in the Milky Way - will be discovered exploiting data from UKIDSs and the upcoming VISTA surveys.

\section{Conclusions}

All participants agreed that the Symposium was interesting and successful. The results presented during the conference boosted lively and important discussions, which will surely result in new collaborations and, in turn, in a greater interest in the field of star cluster research. 